\title{
A very rare case of coexistence of ventricular noncompaction cardiomyopathy, myocardial bridging and atherosclerosis
}

\author{
Ercan Erdogan, Mehmet Akkaya, Ahmet Bacaksiz, Abdurrahman Tasal, Emrah Sevgili \\ Department of Cardiology, Faculty of Medicine, Bezmialem Foundation University, Istanbul, Turkey
}

Postep Kardiol Inter 2013; 9, 2 (32): 176-178

DOI: $10.5114 /$ pwki.2013.35455

\begin{abstract}
Noncompaction of the ventricular myocardium is a rare congenital heart disease, presumably caused by the intrauterine arrest of the myocardial compaction process at the beginning of fetal development. It could remain asymptomatic or manifest with congestive heart failure, arrhythmias, and systemic thromboemboli. Here we report a 55-year-old man who was admitted to hospital with chest pain and dyspnea, whose further evaluation revealed left ventricular noncompaction cardiomyopathy accompanying myocardial bridging and stenosis of the right coronary artery.
\end{abstract}

Key words: ventricular noncompaction, myocardial bridging, atherosclerosis.

\section{Introduction}

Noncompaction of the ventricular myocardium (NVM) is an uncommon congenital cardiomyopathy that is characterized by trabeculations and recesses within the ventricular myocardium, most commonly affecting the left ventricle (LV). It develops as a result of pathological myocardial development during embryogenesis. Although it is often associated with other congenital cardiac defects, it could be seen in the absence of other cardiac anomalies. Early diagnosis allows prompt treatment of the disease which could differ from other causes of cardiomyopathy and lead to a better prognosis, so it is important to recognize this rare cause of heart failure.

\section{Case report}

A 55-year-old man was admitted to the emergency room with new onset chest pain and dyspnea. The 12-lead electrocardiogram showed sinus rhythm with left bundle branch block morphology. Chest examination showed bibasilar rales and decreased breath sounds in both lower lungs. There was bilateral pretibial edema and chest $X$-ray showed mild cardiomegaly.

The patient underwent a transthoracic echocardiogram (TTE) that revealed mild LV systolic dysfunction (LV ejec- tion fraction $=45 \%$ ) with hypokinesis in the inferior septum, inferior and posterior walls. We also noted LV hypertrophy with increased trabecularization and deep intertrabecular spaces in mid segments of the LV anterior and inferior walls, which were compatible with LV noncompaction. The ratio of the noncompacted to the compacted layer measured at end-systole was greater than two. The color Doppler study showed multiple deep intertrabecular recesses filled with blood from the ventricular cavity (Figures $1 \mathrm{~A}, \mathrm{~B}$ ).

Since the patient had chest pain and dyspnea accompanied by wall motion abnormality on TTE, we performed a diagnostic coronary angiogram after the patient became stable. A $90 \%$ discrete lesion in the right coronary artery (RCA) before and after the crux and myocardial bridging in the posterior descending artery were detected (Figures $2 \mathrm{~A}, \mathrm{~B}$ ). Multidetector computed tomographic (MDCT) views illustrate increased trabecularization of the left ventricle with deep recesses that communicate with the ventricular cavity (Figure $3 \mathrm{~A}$ ) and myocardial bridging (MB) in the posterior descending artery (Figure $3 \mathrm{~B}$ ).

\section{Discussion}

Although reported to be $0.05 \%$ in the general population based on echocardiographic studies, the exact preva-

\section{Corresponding author:}

Ercan Erdogan MD, Department of Cardiology, Bezmialem Foundation University, Vatan St, 34093 Istanbul, Turkey, tel.: +90 (212) 453 17 00 ,

e-mail: drercanerdogan@gmail.com

Received: 10.12.2012, accepted: 22.04.2013. 

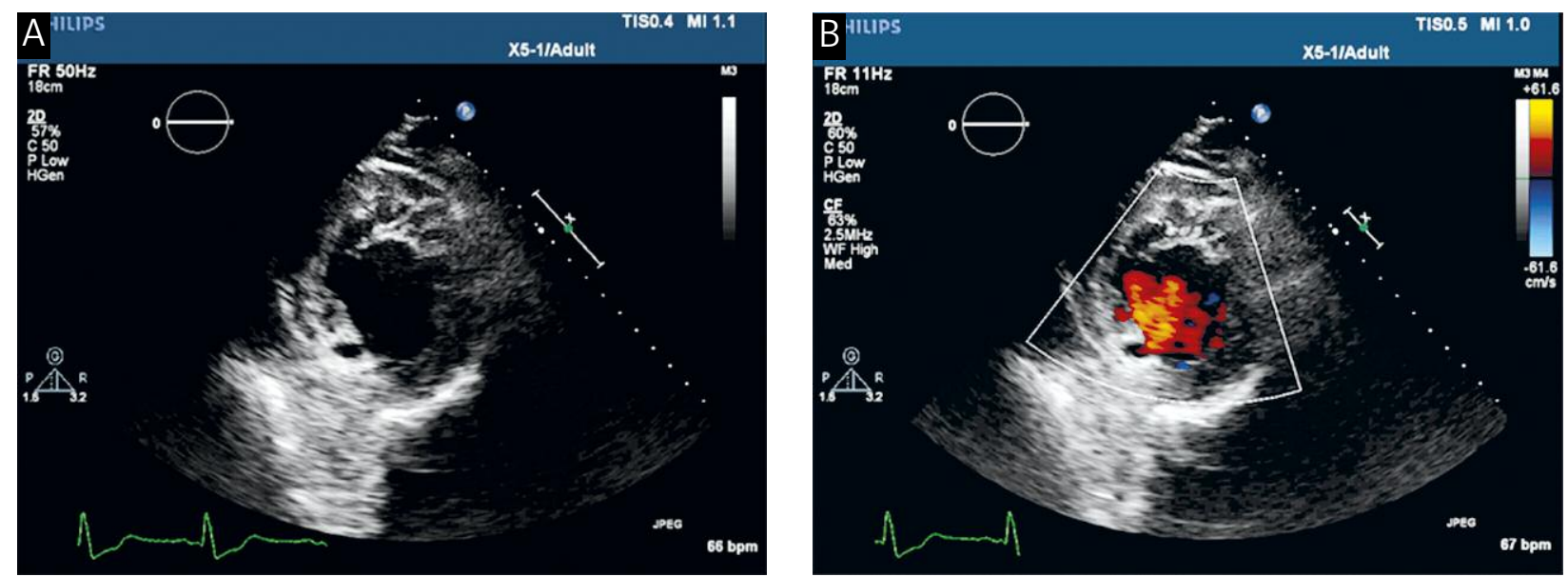

Fig. 1. Transthoracic echocardiogram in parasternal short axis view demonstrating increased trabecularization and deep intertrabecular spaces in mid segments of the left ventricular anterior and inferior walls. The color Doppler study showed multiple deep intertrabecular recesses filled with blood from the ventricular cavity (A, B)
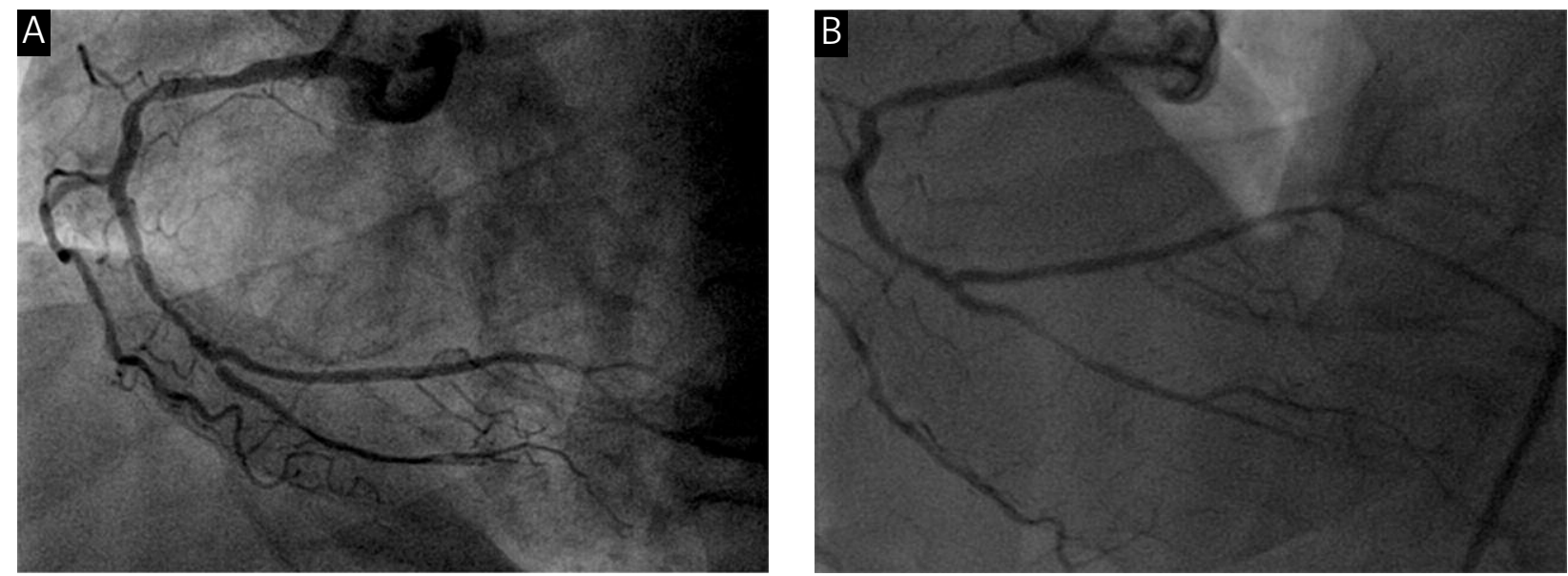

Fig. 2. Coronary angiogram in the left lateral oblique view showing a $90 \%$ discrete lesion in the right coronary artery before and after the crux (A) and myocardial bridging in the posterior descending artery (B)
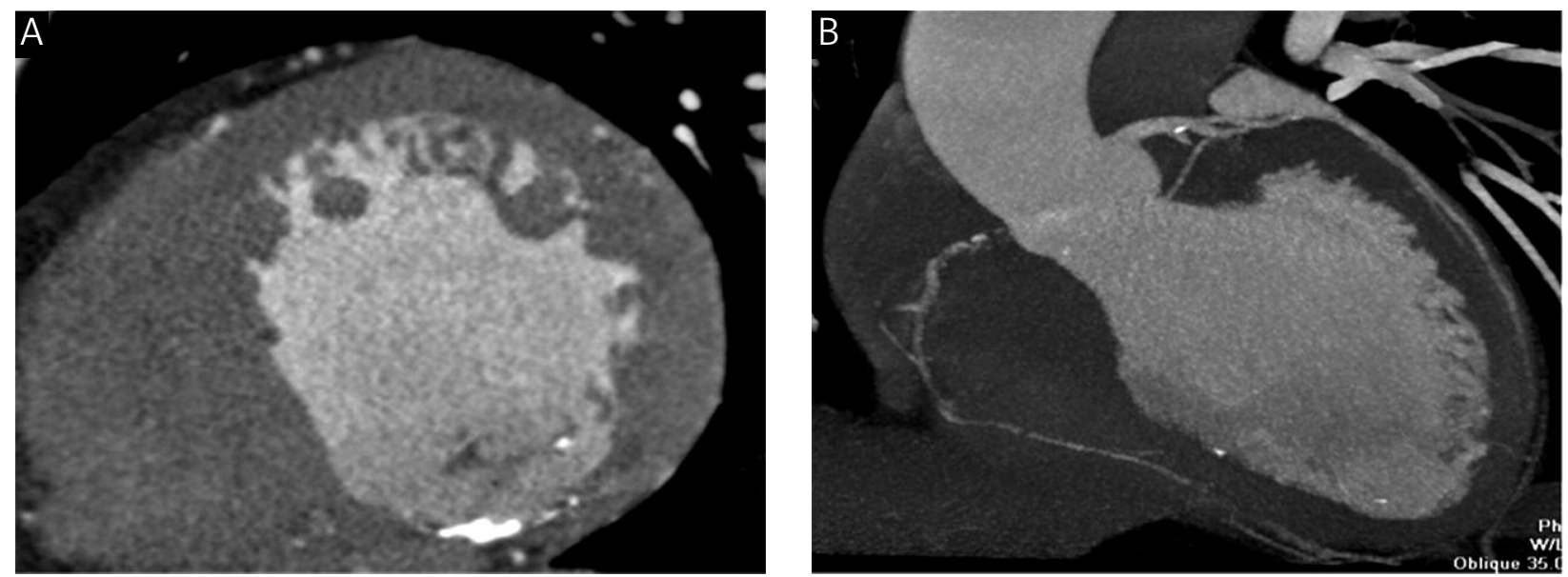

Fig. 3. Short axis multidetector computed tomographic (MDCT) views illustrating increased trabecularization of the left ventricle with deep recesses that communicate with the ventricular cavity (A) and myocardial bridging in the posterior descending artery (B) 
lence of NVM is not known [1]. It affects both genders equally. It is characterized by trabeculations and recesses within the ventricular myocardium, most commonly affecting the LV as an uncommon type of genetic cardiomyopathy [2]. It could also affect the right ventricle in less than half of the cases [3]. The clinical manifestation of this disease usually includes heart failure, rhythm disturbances and cardioembolic events from thrombus formation [4].

Echocardiography is a reliable, noninvasive imaging technique in the diagnosis of patients with NVM; however, patients are often misdiagnosed. On the other hand, over-diagnosis has been reported especially among patients with hypertrophic cardiomyopathy [5]. Both familial and non-familial forms have been reported in the literature and adult patients with NVM seem to be more common than pediatric cases. Also, several genes associated with NVM have been identified. There are some differences between adults and children with NVM. Adults often also have Wolff-Parkinson-White syndrome and complete bundle branch blocks, while children occasionally have concurrent dysmorphic features [6].

Chest pain is not a common symptom in patients with NVM. Most of these patients presenting with angina have a normal coronary angiogram. The angina is predominantly due to chronic myocardial ischemia caused by decreased coronary flow and impaired microvascular function [2].

Myocardial bridge is a congenital condition in which a segment of an epicardial coronary artery runs intramurally through the myocardium. The anterior interventricular branch of the left coronary artery has been reported as the most frequent site of MBs. It predisposes to the development of atherosclerosis in the segment of the coronary artery proximal to the bridge with its shear stress effect [7], revealing that an MB should be viewed as an anatomic risk factor in the assessment of coronary artery disease [8].

In conclusion, coexistence of noncompaction and atherosclerotic coronary artery disease or MB is very rare. To our best knowledge, we report herein the first case of NVM associated with both atherosclerotic narrowing and myocardial bridging.

\section{Acknowledgments}

Published with the written consent of the patient. The authors would like to thank Prof. Omer Goktekin for all his input and guidance on this case report.

\section{References}

1. Espinola-Zavaleta N, Soto M, Castellanos L, et al. Non-compacted cardiomyopathy: clinical-echocardiographic study. Cardiovasc Ultrasound 2006; 4: 35

2. Maron B, Towbin J, Thiene G, et al. Contemporary Definitions and Classification of the Cardiomyopathies: An American Heart Association Scientific Statement From the Council on Clinical Cardiology, Heart Failure and Transplantation Committee; Quality of Care and Outcomes Research and Functional Genomics and
Translational Biology Interdisciplinary Working Groups; and Council on Epidemiology and Prevention. Circulation 2006; 113: 1807-1816.

3. Moric-Janiszewska E, Markiewicz-Loskot G. Genetic heterogeneity of left ventricular noncompaction cardiomyopathy. Clin Cardiol 2008; 31: 201-204.

4. Agmon Y, Connolly HM, Olson LJ, et al. Noncompaction of the ventricular myocardium. J Am Soc Echocardiogr 1999; 12: 859-863.

5. Roberts WC, Katria SJ, Ko JM, et al. Examination of isolated ventricular noncompaction (hypertrabeculation) as a distinct entity in adults. Am J Cardiol 2011; 108: 747-752.

6. Jenni R, Oechslin E, van der Loo B. Isolated ventricular noncompaction of the myocardium in adults. Heart 2007; 93: 11-15.

7. Ge J, Erbel R, Gorge G, et al. High wall shear stres proximal to myocardial bridging and atherosclerosis: intracoronary ultrasound and pressure measurements. Br Heart J 1995; 73: 462-465.

8. de Winter RJ, Kok WE, Piek JJ. Coronary atherosclerosis within a myocardial bridge, not a benign condition. Heart 1998; 80: 91-93. 